\title{
EVALUATION OF FILM-SUBSTRATE ADHESION VIA IMPACT USING COATED BULLETS
}

\author{
Chen-Wu Wu, Zhi-Lin Wu, Kun Zhang and Guang-Nan Chen
}

\begin{abstract}
A method was devised to evaluate the adhesion between a film and a substrate. A front-end coated bullet is accelerated by a gas gun and hits the substrate of the specimen under test. The impact generates a compressive stress pulse that propagates toward the film. After transmission through the interface, part of the pulse is reflected on the free surface of the film, and tensile stress arises at the film-substrate interface, possibly inducing debonding of the film.

This dynamic process was demonstrated analytically and simulated numerically by the finite element method. The results validate the initial concept and lay the foundation for further optimization of this method.
\end{abstract}

\section{Introduction}

Films and coatings with resistance to wear, corrosion, thermal degradation, and other important functions have been adopted in a great many fields [Freund and Suresh 2003]. However, their prospective applications are restricted because of premature debonding. Numerous ways have been tried to evaluate the adhesion between the film and the substrate [Hsueh 2002]. The key step in these experiments is to exert a simple loading directly at the interface and separate the film from the substrate. This is hard to accomplish, especially when the film is strongly bonded. Although some methods, such as scratching and indenting, have been adopted in some situations, the severe deformation around the interface makes it difficult to quantify the adhesion.

The method of laser spallation was developed to overcome this problem [Vossen 1978; Gupta et al. 1990; Gupta and Yuan 1993]. By irradiating the substrate with a laser beam, a compressive stress pulse of short duration is generated. Tensile stress arises after the compressive stress pulse is reflected on the free surface of the film being tested. This tensile stress can peel the film from the substrate if the pulse is strong enough.

It is recognized that the method of laser spallation can exert pulse loadings of short duration directly on the interface and obtain a high strain rate to restrain the effect of plasticity of the materials. At the same time, this dynamic method may reveal some dynamic behaviors of the film-substrate interface that are not attainable by quasistatic methods. However, the laser spallation inevitably involves complicated phenomena such as rapid melting and ablation of the materials, which brings about great difficulties in analysis and modeling.

Keywords: adhesion, film, substrate, coated bullet, impact.

This research is funded by the National Natural Science Foundation of China under grant no. 50531060. 


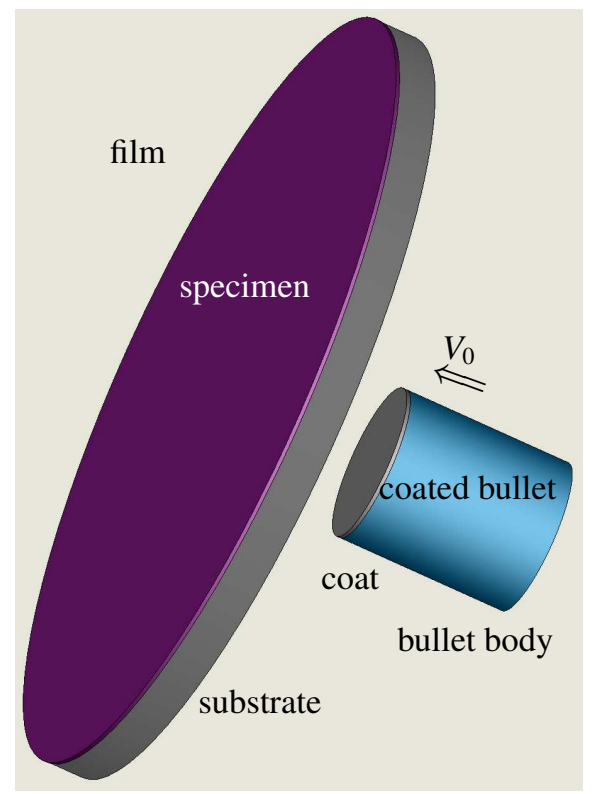

Figure 1. Sketch of the coated bullet and the specimen.

In this study, a method of impact with coated bullets was devised in order to evaluate the interface adhesion between film and substrate. Finite element simulation was then carried out to compute the profiles of the interface stresses as well as the surface stresses of the specimen under impact.

\section{Theoretical formulation of the impact method}

As shown in Figure 1, a cylindrical bullet with a coated front-end is accelerated by a gas gun and hits the substrate of the test specimen, with initial velocity $V_{0}$. Under the impact, a compressive stress pulse is generated and propagate toward the film. Tensile stress then arises after the compressive stress pulse is reflected on the free surface of the film.

For convenience, the coating material for the front-end of the bullet body is chosen to have the same acoustic impedance as the substrate of the specimen. The acoustic impedance of the bullet body is denoted by $\rho_{1} c_{1}$, the acoustic impedance of both the bullet coating and the test substrate is denoted by $\rho_{2} c_{2}$, and the acoustic impedance of the test film is denoted by $\rho_{3} c_{3}$.

To produce an appropriate stress pulse, $\rho_{1} c_{1}$ should be much less than $\rho_{2} c_{2}$, so as to significantly reduce the transmission of the initial compressive stress pulse into the bullet body. In the following, $r$ indicates the radius of the bullet, $l$ is its length, $t_{c}$ the thickness of the coating on the bullet body, $r_{s}$ the radius of the specimen, $t_{s}$ the thickness of the substrate, and $t_{f}$ that of the film. If the equation $t_{c} c_{3}=t_{f} c_{2}$ is satisfied, the spatial width of the stress pulse running through the tested film is $2 t_{f}$. This ensures that the tensile stress arises mainly around the interface in the test.

The propagation and evolution of the stress wave are sketched in Figure 2. At $t=t_{1}$, the coated end of the bullet collides with the substrate and compressive stress waves arise within both the bullet coat and the substrate. At $t=t_{2}$, the compressive stress wave propagating into the bullet impinges on the interface 
of the bullet coat and the bullet body. This wave is partly transmitted into the bullet body and partly reflected, as depicted under $t=t_{3}$. Thus, a compressive stress pulse with a tail develops and propagates toward the specimen, as depicted under $t=t_{4}, t_{5}, t_{6}$.

If $l$ is large enough, the compressive stress pulse $\sigma$ imported into the specimen can be roughly predicted by one-dimensional stress wave theory. The shape of this pulse is seen in Figure 2, right, before the influences of the far rear of the bullet body can be sensed. This prediction is rather accurate, especially in the initial stage after impact; see [Wang 2005, pp. 29-47] for details.

The intensity of the tail of the compressive stress pulse (the plateau in Figure 2, right) is given by $2 \rho_{1} c_{1} \sigma_{0} /\left(\rho_{1} c_{1}+\rho_{2} c_{2}\right)$. This is the stress transmitted into the bullet body. This tail can be decreased to a very small value if $\rho_{1} c_{1}$ is much less than $\rho_{2} c_{2}$. When the input compressive stress pulse impinges on the interface between the film and the substrate in the test $\left(t=t_{7}\right)$, it is, again, partly reflected and partly transmitted into the specimen film [Wang 2005, pp. 29-47]. The reflected and transmitted pulses have intensities, respectively,

$$
\sigma_{r 1}=\frac{\rho_{3} c_{3}-\rho_{2} c_{2}}{\rho_{2} c_{2}+\rho_{3} c_{3}} \sigma \quad \text { and } \quad \sigma_{t}=\frac{2 \rho_{3} c_{3}}{\rho_{2} c_{2}+\rho_{3} c_{3}} \sigma .
$$
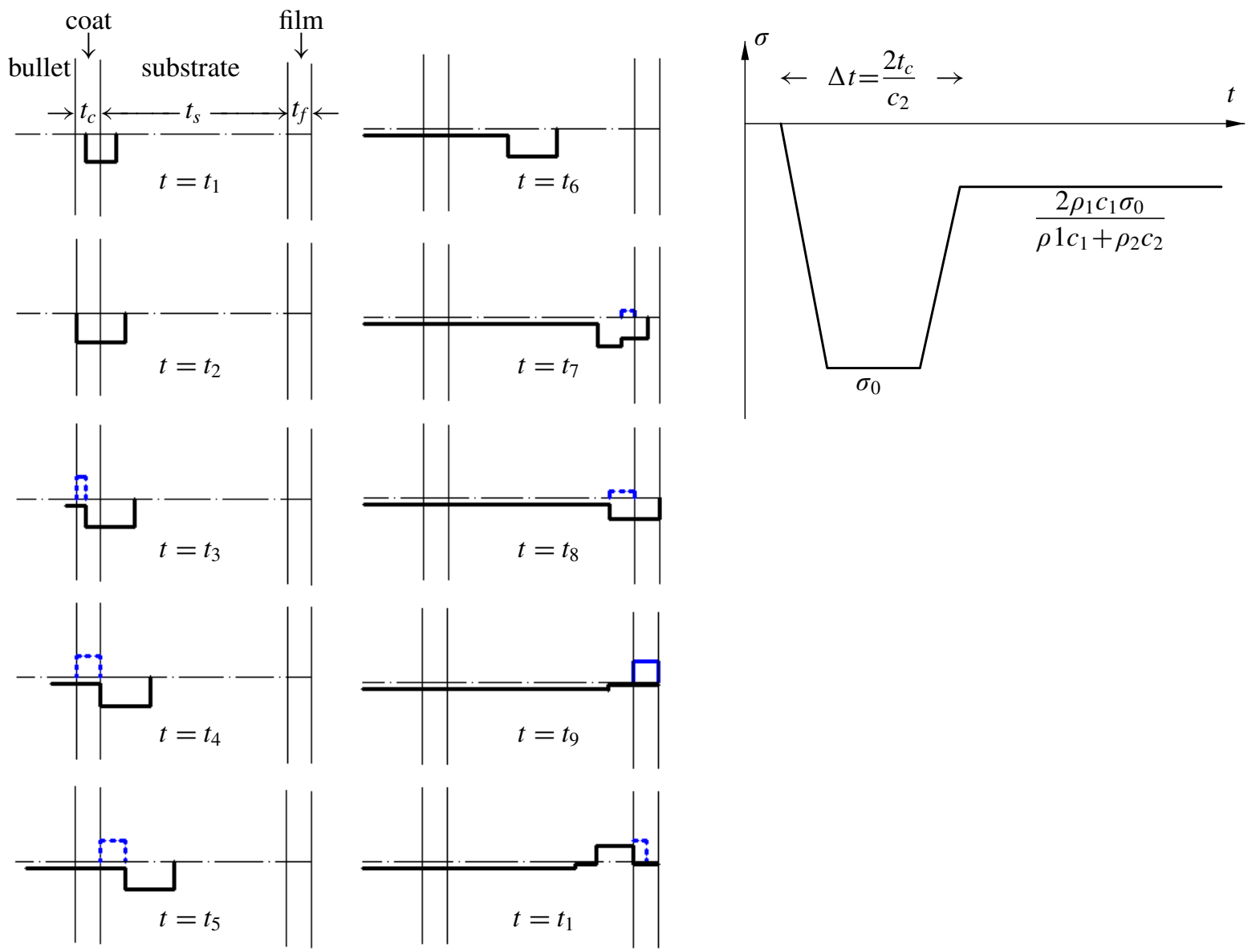

Figure 2. Evolution of the stress wave under impact. 
A tensile stress pulse $\sigma_{r 2}=-\sigma_{t}$ arises after the transmission pulse $\sigma_{t}$ is reflected from the free surface of the film $\left(t=t_{8}, t_{9}\right.$ in Figure 2, left). By taking the algebraic sum of this tensile stress with that of the local tail of the input compressive stress pulse, a resultant tensile stress is obtained around the interface $\left(t=t_{10}\right.$ in Figure 2). If the resulting tensile stress exceeds the bonding strength of the interface, debonding occurs. By measuring the initial velocity of the bullet, for which classical methods of high accuracy can be applied, the interface stress history can be very accurately predicted with the finite element method. Combined with the fracture features of the interface through the experimental observation, the relationship between the interface stress history and the adhesion between the film and the substrate can be established.

\section{Numerical analysis of the stress}

To describe the stress profiles more accurately, an axisymmetric finite element model was set up as shown in Figure 3, and the impact was simulated with the finite element code LS-DYNA. The materials are assumed to be ideally elastic and the parameters chosen are listed in Table 1. Item 1 represents the material of the bullet body, item 2 represents the coating on the bullet and the substrate of the specimen in the test, and item 3 represents the film of the specimen. The geometric parameters are $l=5 \mathrm{~mm}$, $r=2.5 \mathrm{~mm}, t_{s}=1 \mathrm{~mm}$, and $r_{s}=10 \mathrm{~mm}$. A thickness of $t_{c}=t_{f}=0.1 \mathrm{~mm}$ was adopted considering that the acoustic impedance of the coating on the bullet was very near to that of the tested film. The initial velocity of the bullet was $V_{0}=250 \mathrm{~m} / \mathrm{s}$.

We consider the stress component $\sigma_{y y}$ (normal stress) acting on the surface of the substrate and the stress component $\sigma_{y y}$ acting on the interface between the film and the substrate. Figure 4 shows the mean value of $\sigma_{y y}$ over the circular region of radius $r$ around the symmetry axis. It can be seen that the

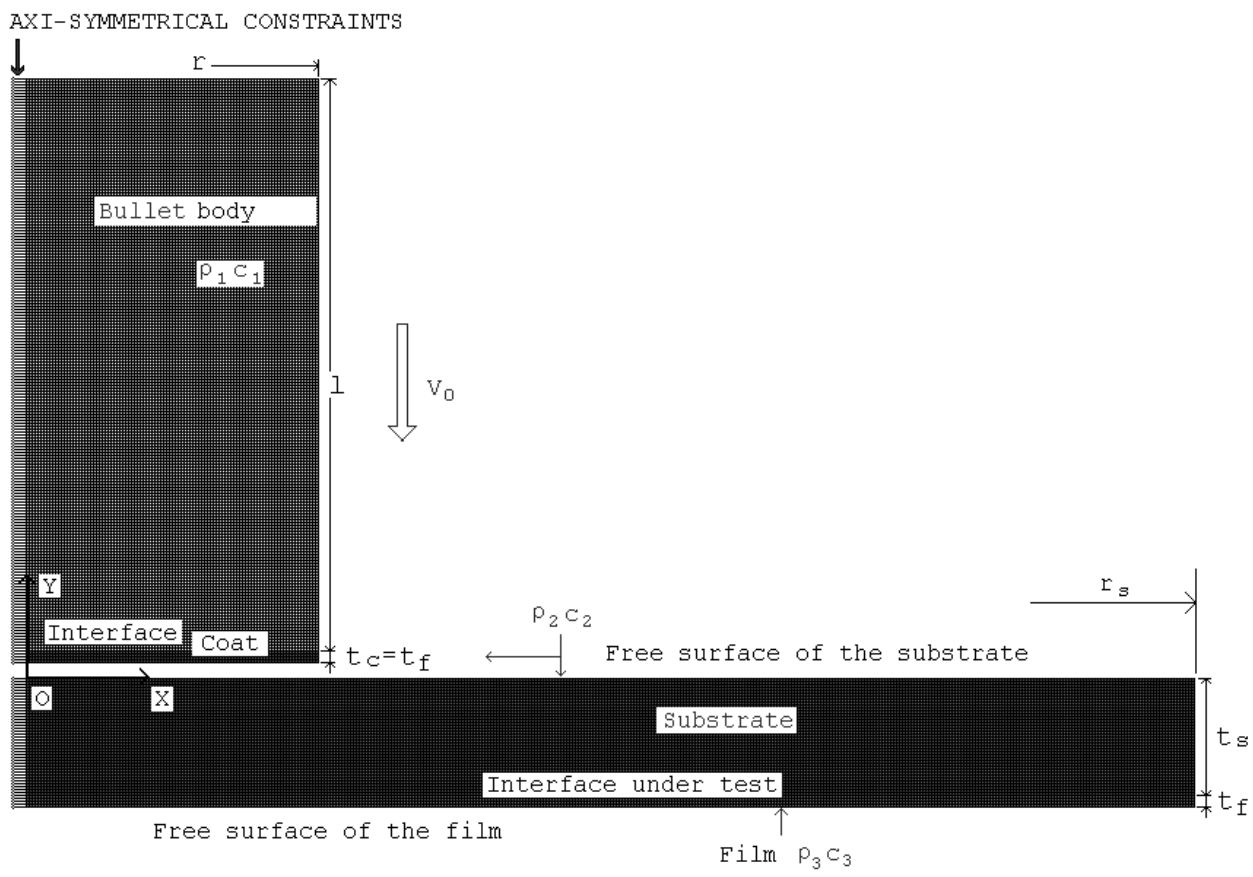

Figure 3. Finite element model of the coated bullet and specimen. 


\begin{tabular}{lccc}
\hline & $\rho\left(\mathrm{kg} / \mathrm{m}^{3}\right)$ & $E(\mathrm{GPa})$ & $v$ \\
\hline $1=$ bullet body & 1050 & 3.38 & 0.35 \\
$2=$ bullet coating, specimen substrate & 7850 & 209 & 0.30 \\
$3=$ film & 7190 & 242 & 0.15 \\
\hline
\end{tabular}

Table 1. Mechanical parameters of the material. $\rho=$ density, $E=$ elastic modulus, $v=$ Poisson's ratio.

input compressive stress pulse (black line in Figure 4) oscillates around the theoretical trend line drawn in Figure 2, right (here represented by the red dots). At the interface, there is a compressive stress pulse and subsequently a tensile stress pulse, both with duration of approximately $t_{f} / c_{3}$. The maximum interface normal stress appears at the instant $t \approx 0.268 \mu \mathrm{s}$. The stress component $\sigma_{y y}$ within the specimen for this instant is shown in Figure 5, in which a thin strip area, close to $r$ in length and adjacent to the interface, is shaded according to the tensile stress.

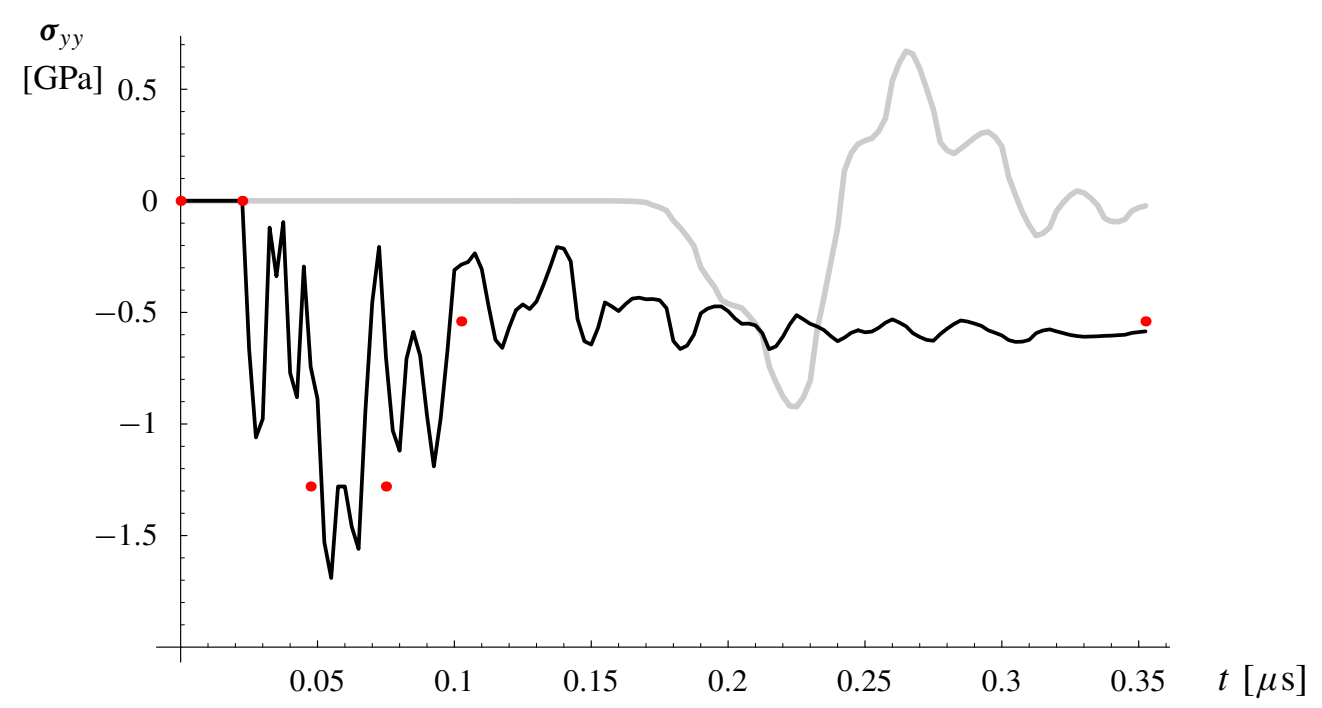

Figure 4. Average normal stress at the surface of the substrate (black line) and at the interface (gray line) as a function of time. The red dots indicate the trend line (pulse shape in Figure 2, right).

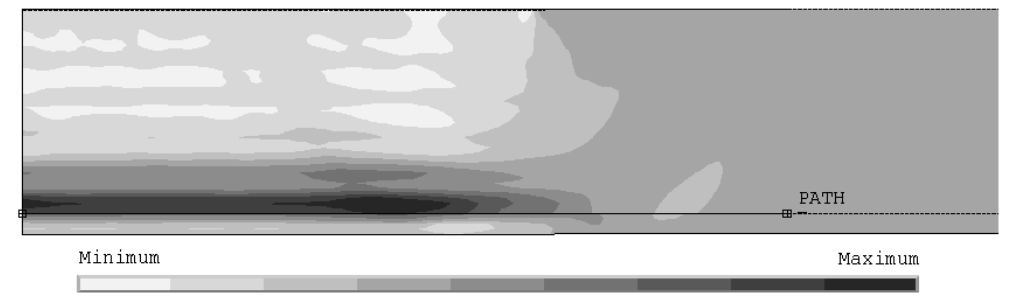

Figure 5. Stress component $\sigma_{y y}$ at $t \approx 0.268 \mu \mathrm{s}$. 


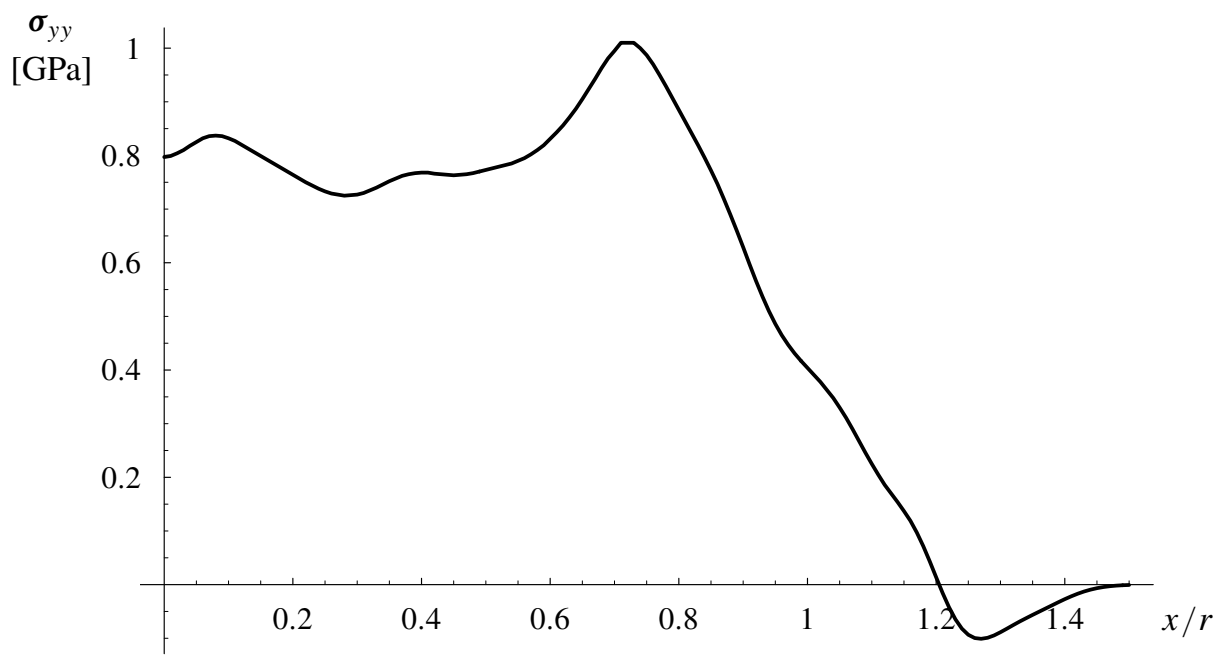

Figure 6. Interfacial normal stress at $t \approx 0.268 \mu \mathrm{s}$.

The interface normal stresses are further mapped at a path of length $1.5 \mathrm{r}$ along the interface as shown in Figure 6, of which the origin is located at the symmetrical axis. This figure suggests that the tensile stress is mainly confined to the circular region of radius $r$ and roughly uniform within that region. It is this tensile stress that can induce the debonding of the film from the substrate.

As mentioned above, the matching of the acoustic impedance of the materials for the bullet body and the bullet coating significantly influences the input compressive pulse. Moreover, the bullet might penetrate the specimen once its kinetic energy exceeds some magnitude [Wang 2005, pp. 29-47]; this is to be avoided in the test. Therefore, in order to produce the expected interface stress and separate the film from the substrate without the bullet penetrating into specimen, an analysis and numerical simulation should be conducted to decide the appropriate range of the initial velocity for specific material pairs.

\section{Conclusions}

In this study, a method of impact with a front-end coated bullet was devised to evaluate the adhesion between a film and a substrate. The evolution of the stress wave during the impact was investigated theoretically. The stresses were calculated numerically. The results showed that a compressive stress pulse with a tail develops on the surface of the substrate. After the compressive stress pulse is reflected on the free surface of the film, tensile stress arises around the interface in the test. This model can be used directly for further optimization of the impact method.

\section{References}

[Freund and Suresh 2003] L. B. Freund and S. Suresh, Thin film materials, Cambridge University Press, Cambridge, 2003.

[Gupta and Yuan 1993] V. Gupta and J. Yuan, "Measurement of interface strength by the modified laser spallation technique, II: Applications to metal/ceramic interfaces”, J. Appl. Phys. 74 (1993), 2397-2404.

[Gupta et al. 1990] V. Gupta, A. S. Argon, J. A. Cornie, and D. M. Parks, "Measurement of interface strength by laser-pulseinduced spallation", Mater. Sci. Eng. A 126 (1990), 105. 
[Hsueh 2002] C. H. Hsueh, "Devising a method to measure film adhesion through modeling", Appl. Phys. Lett. 81:9 (2002), $1615-1617$.

[Vossen 1978] J. L. Vossen, "Measurement of film-substrate bond strength by laser spallation", pp. 122-133 in Adhesion measurement of thin films, thick films, and bulk coatings, edited by K. L. Mittal, ASTM Special Technical Publication 640, American Society for Testing and Materials, Philadelphia, 1978.

[Wang 2005] L. L. Wang, Foundations of stress waves, 2nd ed. ed., National Defense Industry Press, Beijing, 2005. In Chinese.

Received 2 Nov 2008. Revised 2 Apr 2009. Accepted 2 Jun 2009.

CHEN-WU WU: wCw_on@yahoo.com.cn

Institute of Mechanics, Chinese Academy of Sciences, 15 Beisihuanxi Road, Beijing 100190, China

ZHI-LIN WU: wuruinan-1994@mail.njust.edu.cn

School of Mechanical Engineering, Nanjing University of Science and Technology, Nanjing 210094, China

KUN ZHANG: kzhang@imech.ac.cn

Institute of Mechanics, Chinese Academy of Sciences, 15 Beisihuanxi Road, Beijing 100190, China

GUANG-NAN CHEN: gnchen@imech.ac.cn

Institute of Mechanics, Chinese Academy of Sciences, 15 Beisihuanxi Road, Beijing 100190, China 